TAIWANESE JOURNAL OF MATHEMATICS

Vol. 19, No. 1, pp. 101-122, February 2015

DOI: $10.11650 /$ tjm.19.2015.3901

This paper is available online at http://journal.taiwanmathsoc.org.tw

\title{
ON THE REGULARIZED IMAGINARY DOI-NAGANUMA LIFTING
}

\author{
Seokho Jin* and Subong Lim
}

\begin{abstract}
Asai and Friedberg studied the imaginary Doi-Naganuma lifting which sends elliptic modular forms to automorphic forms over an imaginary quadratic field. In this paper we extend this lifting to weak Maass forms by using regularized integral. We construct an automorphic object with singularities on the quadratic upper half-plane $\mathfrak{H}^{1}$ by the regularized theta lifting of a weak Maass form. We also give the convergence region and describe its singularity type. Finally we compute the Fourier coefficients of the lifted form explicitly and present the case of Poincaré series as an example.
\end{abstract}

\section{INTRODUCTION}

A theta lifting gives a correspondence between automorphic forms associated to two groups by the integration with a suitably constructed theta kernel of two variables. A theta lifting has been an important tool in studying automorphic forms by forming connections between different types of automorphic forms. For example, Katok and Sarnak [16] used theta liftings to prove the relation between the central values of the $L$-series of Maass cusp forms to the Fourier coefficients of counterparts under the Shimura correspondence. Theta liftings were also used to study generating series in arithmetic and differential geometry (see $[17,18,19])$.

These classical theta liftings were mainly focused on cusp forms. But there are many interesting researches about weak forms which have possible singularities at cusps (for example, see $[3,24,25]$ ). Therefore it is natural to consider and study the theta lifting of weak forms including weak Maass forms. Borcherds [2] used a regularized theta lifting to get a remarkable product expansions of automorphic forms for $O(p, 2)$. This regularization, which was introduced by Harvey and Moore [13], allows us to overcome the convergence issue and extend theta liftings to weak forms. For related works, see $[5,6,7,23]$.

Received October 14, 2013, accepted May 9, 2014.

Communicated by Yi-Fan Yang.

2010 Mathematics Subject Classification: Primary 11F41; Secondary 11F27.

Key words and phrases: Modular form, Imaginary Doi-Naganuma lifting, Regularized theta integral.

*Corresponding author. 
Our goal is to extend the imaginary Doi-Naganuma lifting to weak Maass forms using the regularized integral. The imaginary Doi-Naganuma lifting is a map from elliptic modular forms to automorphic forms over an imaginary quadratic field, introduced by Asai [1] and Friedberg [10,11]. This kind of lifting was first studied by Doi and Naganuma as a precursor of the base change lifting (see $[9,20]$ ). In this paper we construct an automorphic object with singularities on the quadratic upper half-plane $\mathfrak{H}^{1}$ by the regularized theta lifting of a weak Maass form. To obtain this result, we study the convergence of the regularized theta lifting and show that the lifted form is an eigenfunction of the Casimir operator. We also give the convergence region and describe its singularity type. Finally we compute the Fourier coefficients of the lifted form explicitly and present the case of Poincare series as an example.

From now on we try to explain the results more precisely. Let $k$ be a natural number and $f, g$ functions on the upper half plane $\mathfrak{H}$ such that $\left(\left.f\right|_{k} \sigma\right)(z)=f(z)$, $\left(\left.g\right|_{k} \sigma\right)(z)=g(z)$ for all $\sigma \in \Gamma(N)$. We define

$$
\int_{\Gamma(N) \backslash \mathfrak{H}}^{r e g} f(z) \overline{g(z)} y^{k-2} d x d y:=\int_{S L_{2}(\mathbb{Z}) \backslash \mathfrak{H}}^{r e g} \sum_{\gamma \in \Gamma(N) \backslash S L_{2}(\mathbb{Z})}\left(\left.f\right|_{k} \gamma\right)(z) \overline{\left(\left.g\right|_{k} \gamma\right)(z)} y^{k-2} d x d y,
$$

where $\int_{S L_{2}(\mathbb{Z}) \backslash \mathfrak{H}}^{r e g}$ is the regularized integral in the sense of Borcherds (see [2, section 6] for the definition) and $z=x+i y \in \mathfrak{H}$. In this paper we use this to define the regularized Doi-Naganuma lifting. We are given a quadratic lattice $\Lambda$ of signature $(3,1)$ arising from an imaginary quadratic field $K$ and an element $V$ of the dual lattice of $\Lambda$. The regularized lifting of a weak Maass form $f$ of weight $k$ associated to $\Lambda$ and $V$ is given by $F^{r e g}(g, V, \Lambda ; f)={ }^{t}\left(F_{1-k}^{r e g}, \cdots, F_{k-1}^{r e g}\right)$, where for $|\alpha| \leq k-1$ the component $F_{\alpha}^{r e g}(g, V, \Lambda ; f)$ is defined by

$$
F_{\alpha}^{r e g}(g, V, \Lambda ; f):=\int_{\Gamma(N) \backslash \mathfrak{H}}^{r e g} \theta_{k, \alpha}(z, V, g, \Lambda) \overline{f(z)} y^{k-2} d x d y .
$$

Here, ${ }^{t} A$ denotes the transpose of $A$ and $\theta_{k, \alpha}(z, V, g, \Lambda)$ is a theta function of variables $z \in \mathfrak{H}$ and $g \in S L_{2}(\mathbb{C})$. Then it turns out that the result is a weak Maass form for $K$ of weight $2 k-2$ for $g=g(\mu, \nu)=\left(\begin{array}{cc}\nu^{1 / 2} & \mu \nu^{-1 / 2} \\ 0 & \nu^{-1 / 2}\end{array}\right)$, when $\mu+\nu \mathbf{k} \in \mathfrak{H}^{1}$ (Theorem 3.2).

It is also important to compute the Fourier expansions of the regularized theta lifting and study its singularities. We compute the singularity type in Theorem 4.1 and the Fourier expansion in Theorem 4.2 and Theorem 4.3.

In [10] Friedberg gave an interesting example showing that the lifted form of the non-holomorphic Eisenstein series of weight $k>1$ is a Poincaré type series. The following example shows that the same phenomenon happens in the case where $f$ is a weakly holomorphic Poincare series. Let $\psi$ be a negative integer. Then it is known (see [15]) that the Poincare series

$$
f_{\psi}(z):=\sum_{\gamma \in \Gamma_{\infty} \backslash \Gamma(N)} \frac{e^{2 \pi i \psi \gamma z}}{(c \tau+d)^{k}}
$$


vanishes at all cusps of $\Gamma(N)$ except possibly at $i \infty$, and at $i \infty$ it has a Fourier expansion of the form

$$
f_{\psi}(z)=e^{2 \pi i \psi z}+\sum_{m>0} a_{m}(\psi) e^{2 \pi i m z} .
$$

Then the regularized lifting $F^{r e g}\left(g(\mu, \nu) ; g_{\psi}\right)$ is equal to

$$
\sum_{\substack{X \in \Lambda \\ Q[X-V]=2 \psi}} \frac{N \Gamma(k-1 / 2)}{\pi\left(R^{g(\mu, \nu)}[X-V]+\psi\right)^{k-1 / 2}} .
$$

Since the result is absolutely convergent for $k \geq 2$, it can be regarded as a type of Poincaré series.

The remainder of the paper is organized as follows. In section 2 , we deal with preliminary notions. We define weak Maass forms on $\mathfrak{H}$ and $\mathfrak{H}^{1}$ respectively and introduce the theta functions $\theta_{k, \alpha}$ and their properties. This enables us to compute the Fourier expansion of $\theta_{k, \alpha}$ at arbitrary cusps. In section 3 we prove several properties of the regularized theta lifting to prove Theorem 3.2. We compute the convergence region and study the modularity and the behavior of $F_{\alpha}^{r e g}(g(\mu, \nu) ; f)$ under the Casimir operators. Finally in section 4 we compute its singularity type and the Fourier expansion.

\section{Preliminaries}

In this section we set several notations and define basic objects, following [10]. For a function $f(z)$ on $\mathfrak{H}$ and a matrix $\gamma=\left(\begin{array}{ll}a & b \\ c & d\end{array}\right) \in G L_{2}^{+}(\mathbb{R})$, we define the slash operator of integral weight $k$ by $\left(\left.f\right|_{k} \gamma\right)(z)=(\operatorname{det} \gamma)^{k / 2}(c z+d)^{-k} f(\gamma z)$, where $\gamma z=\frac{a z+b}{c z+d}$.

\subsection{Weak Maass Forms}

We start with the definition of a weak Maass form. Let $\Delta_{k}$ be the hyperbolic Laplacian of weight $k$ given by

$$
\Delta_{k}:=-y^{2}\left(\frac{\partial^{2}}{\partial x^{2}}+\frac{\partial^{2}}{\partial y^{2}}\right)+i k y\left(\frac{\partial}{\partial x}+i \frac{\partial}{\partial y}\right) .
$$

Definition 2.1. A weak Maass form of weight $k \in \mathbb{Z}$ on the congruence subgroup $\Gamma \subset S L_{2}(\mathbb{Z})$ and eigenvalue $-\lambda \in \mathbb{C}$ is a smooth function $f: \mathfrak{H} \rightarrow \mathbb{C}$ such that

(1) $\left.f\right|_{k} \gamma=f$ for all $\gamma \in \Gamma$,

(2) $\Delta_{k} f=-\lambda f$

(3) $f(z)$ has at most linear exponential growth at all of the cusps, i.e., $\left(\left.f\right|_{k} \gamma\right)(z)=$ $O\left(e^{\delta y}\right)$ for all $\gamma \in S L_{2}(\mathbb{Z})$, for some $\delta>0$.

We denote the space of weak Maass forms by $W M F_{k,-\lambda}(\Gamma)$. 
Let $\gamma \in S L_{2}(\mathbb{Z})$. It is known that the Fourier expansion for a weak Maass form $\left(\left.f\right|_{k} \gamma^{-1}\right)(z) \in W M F_{k,-\lambda}(\Gamma(N))$ at the cusp corresponding to $\gamma$ is given by

$$
\begin{aligned}
& \left(\left.f\right|_{k} \gamma^{-1}\right)(z) \\
= & \sum_{\substack{n=-n_{\gamma} \\
l_{\gamma}}} \tilde{a}_{\gamma}(n, s)|4 \pi n y / N|^{-\frac{k}{2}} W_{-s g n(n) \frac{k}{2}, s-\frac{1}{2}}(-4 \pi|n| y / N) e^{2 \pi i n x / N} \\
& +\sum_{n \in \mathbb{Z} \backslash\{0\}} a_{\gamma}(n, s)|4 \pi n y / N|^{-\frac{k}{2}} W_{\operatorname{sgn}(n) \frac{k}{2}, s-\frac{1}{2}}(4 \pi|n| y / N) e^{2 \pi i n x / N} \\
& +a_{\gamma}(0, s) y^{s-\frac{k}{2}}+\tilde{a}_{\gamma}(0, s) y^{1-s-\frac{k}{2}},
\end{aligned}
$$

where $n_{\gamma}, l_{\gamma} \in \mathbb{N}, W_{k, m}(y)$ and $W_{-k, m}(-y)$ are the standard W-Whittaker's functions and $-\lambda=s(1-s)+\frac{k^{2}-2 k}{4}$ (for the details see [4]). It is also known that $a_{\gamma}(n, s)=$ $O\left(e^{C \sqrt{n}}\right)$ for some $C>0$ (see $\left.[5,6]\right)$. We define a subspace $W M F_{k,-\lambda}^{+}(\Gamma(N))$ by

$$
W M F_{k,-\lambda}^{+}(\Gamma(N))=\left\{f \in W M F_{k,-\lambda}(\Gamma(N)) \mid \tilde{a}_{\gamma}(n, s)=0 \text { for } n>0\right\} .
$$

\subsection{Weak Maass forms for Imaginary Quadratic Fields}

Now we introduce the space of Hilbert modular forms on the quaternionic upper half space $\mathfrak{H}^{1}=\{\mu+\nu \mathbf{k} \in \mathcal{H}(-1,-1) \mid \mu \in \mathbb{C}, \nu>0\}$, where $\mathcal{H}(-1,-1)$ is the quaternion algebra with the standard basis $1, \mathbf{i}, \mathbf{j}$, and $\mathbf{k}$. Recall that $\sigma=\left(\begin{array}{ll}a & b \\ c & d\end{array}\right) \in$ $\mathbf{G}=S L_{2}(\mathbb{C})$ acts on $z_{1}$ in $\mathfrak{H}^{1}$ by $\sigma z_{1}=\left(a z_{1}+b\right)\left(c z_{1}+d\right)^{-1}$ and that the stabilizer of $\mathbf{k}$ is $S U(2, \mathbb{C})$, the special unitary group. Note that $S U(2, \mathbb{C})$ is not abelian so we need to consider multi-dimensional representations of $S U(2, \mathbb{C})$. For $n \in \mathbb{Z}_{\geq 0}$ let $(a b)_{n}:={ }^{t}\left(a^{n}, a^{n-1} b, \cdots, a b^{n-1}, b^{n}\right)$ and define the $n$-fold symmetric representation $\rho_{n}(g)$, for $g \in S L_{2}(\mathbb{C})$, by $\rho_{n}(g)(a b)_{n}:=\left((a b)^{t} g\right)_{n}$. Then $\rho_{n}$ gives an irreducible $(n+1)$-dimensional representation of $S U(2, \mathbb{C})$.

Let $\rho\left(z_{1}=\mu+\nu \mathbf{k}\right)=\left(\begin{array}{cc}\mu & -\nu \\ \bar{\nu} & \bar{\mu}\end{array}\right)$ for $\mu, \nu \in \mathbb{C}$. For $g=\left(\begin{array}{ll}a & b \\ c & d\end{array}\right) \in \mathbf{G}$ and $z_{1} \in \mathfrak{H}^{1}$ set $J\left(g, z_{1}\right)=\rho\left(c z_{1}+d\right)$ and $\rho_{n}^{1}(g)={ }^{t} \rho_{n}\left({ }^{t} g\right)$. Then we can define a slash operator for a function $F^{1}: \mathfrak{H}^{1} \rightarrow \mathbb{C}^{n+1}$ by

$$
\left(F^{1}||[g]_{n}\right)\left(z_{1}\right):=\rho_{n}^{1}\left(J\left(g, z_{1}\right)\right)^{-1} F^{1}\left(g z_{1}\right)
$$

for all $g \in \mathbf{G}$. We can also extend the slash operator to $A \in G L_{2}(\mathbb{C})$ by putting

$$
F^{1}\left\|[A]_{n}=F^{1}\right\|\left[(\operatorname{det} A)^{-\frac{1}{2}} A\right]_{n}
$$

(as stated in [10, p.491], the ambiguity of sign arising from by the choice of square root will not cause any harm in the applications).

Let $K$ be an imaginary quadratic field, and let $-D$ be its discriminant. 
Definition 2.2. Let $\Xi$ a congruence subgroup of $S L_{2}\left(\mathcal{O}_{K}\right)$. A weak Maass form for $K$ of weight $n$ on $\Xi$ and eigenvalues $\left(\lambda, \lambda^{\prime}\right)$ is a real analytic function $F^{1}: \mathfrak{H}^{1} \rightarrow \mathbb{C}^{n+1}$ with singularities satisfying

(1) $\Psi^{n} F^{1}=\lambda F^{1}$ and $\tilde{\Psi}^{n} F^{1}=\lambda^{\prime} F^{1}$,

(2) $F^{1} \|[\sigma]_{n}=F^{1}$ for all $\sigma$ in $\Xi$,

(3) $\left(F^{1} \|[A]_{n}\right)\left(z_{1}\right)=O\left(\nu^{\delta}\right)$ as $\nu \rightarrow \infty$, uniformly in $x$ for all $A \in G L_{2}\left(\mathcal{O}_{K}\right)$, for some fixed real $\delta$.

Here $\Psi^{n}, \tilde{\Psi}^{n}$ are Casimir operators of weight $n$ on $\mathfrak{H}^{1}$ which are given by

$$
\begin{aligned}
& \Psi^{n}=y^{2}\left(\partial_{x \bar{x}}^{2}+\partial_{y y}^{2} / 4\right) E_{n+1}+y \partial_{x}\left(\begin{array}{ccccc}
0 & 1 & & & \\
& \ddots & 2 & \\
& & \ddots & \ddots & \\
& & & \ddots & \\
& & & & 0 \\
& & & 0
\end{array}\right)
\end{aligned}
$$

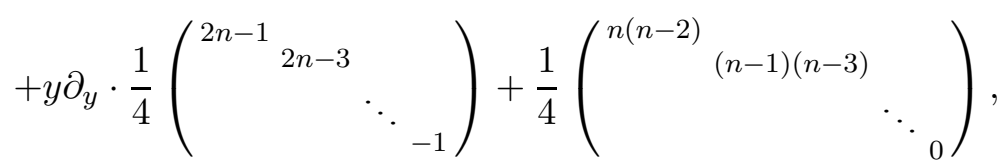

$$
\begin{aligned}
& \tilde{\Psi}^{n}=y^{2}\left(\partial_{x \bar{x}}^{2}+\partial_{y y}^{2} / 4\right) E_{n+1}-y \partial_{x}\left(\begin{array}{ccccc}
0 & & & & \\
& \ddots & & & \\
& \ddots & & & \\
& n-1 & \ddots & & \\
& & \ddots & \ddots & \\
& & & 1 & 0
\end{array}\right)
\end{aligned}
$$

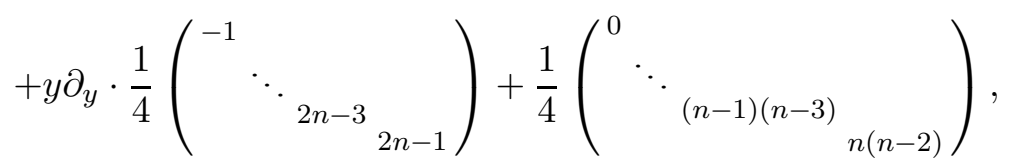

where $E_{n+1}$ is the identity matrix of size $n+1$.

Let $\left\{\left\{\Xi, n,\left\{\lambda, \lambda^{\prime}\right\}\right\}\right\}$ be the space of all these forms. If $F^{1}$ is defined without singularities, then it is said to be a modular form for $K$, and moreover if $F^{1}$ satisfies a better growth condition at infinity, namely for all $A \in G L_{2}\left(\mathcal{O}_{K}\right), F^{1} \|[A]_{n}\left(z_{1}\right)=$ $O\left(e^{-\delta \nu}\right)$ as $\nu \rightarrow \infty$ uniformly in $\mu$, for some fixed $\delta>0, F^{1}$ is said to be cuspidal or a cusp form. If $\lambda=\lambda^{\prime}$, we say simply that $F^{1}$ has eigenvalue $\lambda$.

For later use we recall the following proposition.

Proposition 2.3. ([10, Proposition 2.2]). There is a one-to-one correspondence between

$$
\left\{F: \mathbf{G} \rightarrow \mathbb{C}^{n+1} \mid F(\gamma g \kappa)={ }^{t} \rho_{n}(\kappa) F(g) \text { for all } \gamma \in \Xi, \kappa \in S U(2, \mathbb{C})\right\}
$$


and

$$
\left\{F^{1}: \mathfrak{H}^{1} \rightarrow \mathbb{C}^{n+1} \mid F^{1}\left(\gamma z_{1}\right)=\rho_{n}^{1}\left(J\left(\gamma, z_{1}\right)\right) F^{1}\left(z_{1}\right) \text { for all } \gamma \in \Xi\right\}
$$

given by $F^{1}\left(z_{1}\right)=\rho_{n}^{1}(J(g, \mathbf{k})) F(g)$ and $F(g)=\rho_{n}^{1}(J(g, \mathbf{k}))^{-1} F^{1}(g \mathbf{k})$, where $z_{1}=$ $g \mathbf{k}$.

This correspondence is also compatible with the action of Casimir operators (see [10, section 2]). Also note that for an element $g(\mu, \nu)=\left(\begin{array}{cc}\nu^{1 / 2} & \mu \nu^{-1 / 2} \\ 0 & \nu^{-1 / 2}\end{array}\right)$ in $S L_{2}(\mathbb{C})$, where $\mu \in \mathbb{C}$ and $\nu>0$, the corresponding element in $\mathfrak{H}^{1}$ is $g(\mu, \nu) \mathbf{k}=\mu+\nu \mathbf{k}$.

\subsection{Theta function}

We introduce a quadratic vector space $\mathcal{V}=\left\{\left.X \in M_{2 \times 2}(\mathbb{C})\right|^{t} X=\bar{X}\right\}$ with a symmetric bilinear form $Q(X, Y):=-\operatorname{tr}(X \operatorname{adj}(Y))$ and a quadratic form $Q[X]:=$ $Q(X, X)=-2 \operatorname{det} X$. Here $\operatorname{adj}(Y)$ is the adjoint of $Y$ defined by $\operatorname{adj}\left(\left(\begin{array}{ll}a & b \\ c & d\end{array}\right)\right)=$ $\left(\begin{array}{cc}d & -b \\ -c & a\end{array}\right)$. We remark that $\tilde{Y}=\left(\begin{array}{cc}0 & -1 \\ 1 & 0\end{array}\right) Y\left(\begin{array}{cc}0 & 1 \\ -1 & 0\end{array}\right)$ used in $[1,10]$ is the complex conjugate of $\operatorname{adj}(Y)$ for $Y \in \mathcal{V}$, but $\operatorname{adj} Y$ gives the correct pairing. For $X \in \mathcal{V}$ and $g \in \mathbf{G}$ let $X^{g}:={ }^{t} \bar{g} X g$, and let $R^{g}[X]:=\operatorname{tr}\left(\left(X^{g}\right)^{2}\right)$ be its majorant corresponding to $g$. Given $M, P \in \mathbb{Z}_{\neq 0}$ and an ideal $I$, we introduce the lattice

$$
\Lambda_{i}:=\left\{(\underset{\bar{r}}{m} \underset{p}{m}) \in \mathcal{V} \mid m \in M \mathbb{Z}, p \in P \mathbb{Z}, r \in J_{i}\right\},
$$

where $J_{i}=I \mathfrak{E}_{i} \mathfrak{e}_{i}^{-1}, \mathfrak{E}_{1}=\mathcal{O}_{K}, \cdots, \mathfrak{E}_{h(K)}$ is a set of ideal class representatives, and take $\mathfrak{e}_{i}$ ideal numbers for these ideals in the sense of Hecke (see [14]). Let $N_{1}=$ l.c.m $(M P, \mathbf{N}(I) D)$.

For a fixed $\Lambda=\Lambda_{i}$ let $\Lambda^{*}$ be the dual lattice of $\Lambda$ given by $\Lambda^{*}=\Lambda_{i}^{*}=\{(\underset{r}{r} \underset{p}{r}) \in$ $\left.\mathcal{V} \mid P m \in \mathbb{Z}, M p \in \mathbb{Z}, r \in \bar{J}_{i}^{-1} \mathcal{D}_{K}^{-1}\right\}$, where $\mathcal{D}_{K}^{-1}$ is the inverse different. Then for a vector $V$ in the dual lattice $\Lambda^{*}$, there is a theta series

$$
\theta_{k, \alpha}(z, V, g, \Lambda):=y^{1 / 2} \sum_{X \in \Lambda} \eta_{k, \alpha}\left((X-V)^{g}\right) e\left(\left(x Q+i y R^{g}\right)[X-V]\right)
$$

for $z=x+i y \in \mathfrak{H}, g \in \mathbf{G}$ and $k \in \mathbb{Z}_{\geq 1}$ (see [1]), where $e(x)=e^{\pi i x}$. Here,

$$
\eta_{k, \alpha}(X):=(k-1) ! \sum_{\beta, \gamma} \frac{r^{\alpha} L_{\beta}^{(\alpha)}(2 r \bar{r}) H_{\gamma}(m-p)}{(\alpha+\beta) ! \gamma ! 2^{\beta}},
$$

where $\sum_{\beta, \gamma}$ denotes the sum over $\beta$ and $\gamma$ nonnegative integers such that $2 \beta+\gamma=$ $k-1-\alpha$ and $\alpha+\beta \geq 0$ and $L_{n}^{(\alpha)}$ and $H_{n}$ are Laguerre and Hermite polynomials given by

$$
L_{n}^{(\alpha)}(y)=e^{y}\left(n ! y^{\alpha}\right)^{-1} \frac{d^{n}}{d y^{n}}\left(e^{-y} y^{n+\alpha}\right), H_{n}(y)=(-1)^{n} e^{y^{2} / 2} \frac{d^{n}}{d y^{n}}\left(e^{-y^{2} / 2}\right) .
$$

Note that $\eta_{k, \alpha}(X)$ form a $\mathbb{C}$-basis of spherical harmonic polynomials of degree $k-1$. 
Proposition 2.4. [10, Proposition 3.1]. For all $\sigma=\left(\begin{array}{ll}a & b \\ c & d\end{array}\right) \in \Gamma_{0}\left(N_{1}\right)$

$$
\left(\left.\theta_{k, \alpha}\right|_{k} \sigma\right)(z, d V, g, \Lambda)=\chi_{-D}(d) \theta_{k, \alpha}(z, V, g, \Lambda),
$$

where $\chi_{-D}(n)=\left(\frac{-D}{n}\right)$ and $(-)$ is the Kronecker symbol. In particular, for $\sigma \in$ $\Gamma\left(N_{1}\right)$,

$$
\left(\left.\theta_{k, \alpha}\right|_{k} \sigma\right)(z, V, g, \Lambda)=\theta_{k, \alpha}(z, V, g, \Lambda) .
$$

We write $\operatorname{Tr}_{\mathbb{R}}^{\mathbb{C}}$ for the trace from $\mathbb{C}$ to $\mathbb{R}$ and $\mathbf{N}=\operatorname{Norm}(\mathbb{C} / \mathbb{R})$. Let $\phi_{\alpha, \beta}(z, u, v):=\sum_{r \in J} y^{-\beta} L_{\beta}^{(\alpha)}(4 \pi y \mathbf{N}(r-v))(r-v)^{\alpha} e\left(2 \mathbf{N}(r-v) z+2 \operatorname{Tr}_{\mathbb{R}}^{\mathbb{C}}(r \bar{u})-\operatorname{Tr}_{\mathbb{R}}^{\mathbb{C}}(u \bar{v})\right)$, where $L_{\beta}^{\alpha}(y)$ is the Laguerre polynomial defined in Section 2.3. Then by [11, Lemma 3.5]

$$
\phi_{\alpha, \beta}(\sigma z, a u+b v, c u+d v)=\chi_{-D}(d)(c z+d)^{\alpha+2 \beta+1} \phi_{\alpha, \beta}(z, u, v)
$$

for $\alpha, \beta \geq 0, z \in \mathfrak{H}, u, v \in \mathbb{C}$ and $\sigma \in \Gamma_{0}(D \mathbf{N}(I))$. The definition of $\phi_{\alpha, \beta}$ is motivated by the fact that $\theta_{k, \alpha}$ is the sum of products of functions of $r$ and $\bar{r}$ and functions of $m$ and $p$, with $\phi_{\alpha, \beta}$ being precisely the former (see [11, Lemma 3.6]).

\subsection{Fourier expansion of $\left(\left.\theta_{k, \alpha}\right|_{k} \gamma\right)(z, V, g, \Lambda)$}

In this subsection we briefly explain that $\theta_{k, \alpha}$ can be realized as the theta function with the Weil representation and find the Fourier expansion of $\left(\left.\theta_{k, \alpha}\right|_{k} \gamma\right)(z)$. We follow the notations given in [21].

We briefly recall the first spherical property (for the details see [8]). Let $\varphi \in$ $\mathcal{S}\left(\mathbb{R}^{n}\right)$ and $l \in \mathbb{Z}$. Then $\varphi$ is said to be of first spherical property for weight $\frac{l}{2}$ if

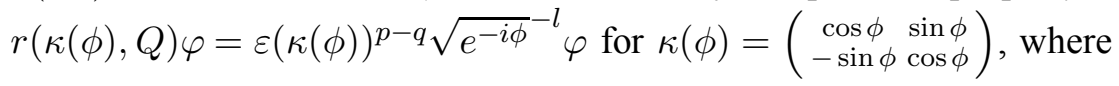

$$
\varepsilon(\sigma)= \begin{cases}\sqrt{i} & c>0 \\ i^{(1-\operatorname{sgn} d) / 2} & c=0 \\ \sqrt{i}^{-1} & c<0\end{cases}
$$

for $\sigma=\left(\begin{array}{ll}a & b \\ c & d\end{array}\right) \in S L_{2}(\mathbb{R})$. Now we introduce the theta series coming from the Weil representation. If we let

$$
\varphi(X)=\eta_{k, \alpha}\left(X^{g}\right) e^{-\pi R^{g}[X]}
$$

for $X \in \mathcal{V}$, then this function clearly is a Schwartz function. In the following we show that this $\varphi$ satisfies the first spherical property of weight $k$.

Let $\eta_{(k)}(X):=\left(\eta_{k, 1-k}(X), \cdots, \eta_{k, k-1}(X)\right)$ and $P_{k-1}(X):=\eta_{(k)}(X)^{t}(a b)_{2 k-2}$. Then we see that $P_{k-1}(X)=Q\left(X, A_{1}\right)^{k-1}$, where $(a b)_{2 k-2}=\left(a^{2 k-2}, a^{2 k-3} b, \cdots\right.$, $b^{2 k-2}$ ) and $A_{1}={ }^{t}(a b)\left(\begin{array}{ll}a & b\end{array}\right)\left({ }_{1}{ }^{-1}\right)$ (see [11, section 1.3]). We let $F_{z}(X):=$ 
$P_{k-1}(X) e^{\pi i Q_{z}(X)}$, where $Q_{z}(X)=x Q[X]+i y R^{I_{2 \times 2}}[X]$ and $z=x+i y \in \mathfrak{H}$. Furthermore, since $Q(A, B)=Q\left(A^{g}, B^{g}\right)$ for all $g \in S L_{2}(\mathbb{C})$, we see $\eta_{(k)}\left(X^{g}\right)^{t}(a b)_{2 k-2}=$ $Q\left(X^{g}, A_{1}\right)^{k-1}=Q\left(X, A_{1}^{g^{-1}}\right)^{k-1}=P_{k-1}\left(X^{g}\right)$. Then

$$
F_{i}\left(X^{g}\right)=P_{k-1}\left(X^{g}\right) e^{-\pi R^{I_{2 \times 2}\left[X^{g}\right]}}=Q\left(X, A_{1}^{g^{-1}}\right)^{k-1} e^{-\pi R^{g}[X]} .
$$

Therefore we can see that $F_{i}\left(X^{g}\right)$ satisfies the first spherical property for weight $k$ by Lemma 1.2 of [21]. Finally, since the Weil representation acts independently of $a, b$, the function $\eta_{k, \alpha}\left(X^{g}\right) e^{-\pi R^{g}[X]}$ is also of the first spherical property for weight $k$. Then $\theta_{k, \alpha}$ coincides with the theta function in [8]: $\theta(z, \varphi,-V)=\theta_{k, \alpha}(z, V, g, \Lambda)$.

From this interpretation we can compute the Fourier expansion of $\left.\theta_{k, \alpha}\right|_{k} \gamma$ by using Theorem 1.5 of [8]. Note that the signature is $(3,1)$ and the weight is $k$ for $\theta_{k, \alpha}$. For $\gamma \in \Gamma$ we get

$$
\left(\left.\theta_{k, \alpha}\right|_{k} \gamma\right)(z, V, g, \Lambda)=i^{-\operatorname{sgn} c} \sum_{X \in \Lambda^{*}} c(-V, X)_{\gamma} y^{1 / 2} \eta_{k, \alpha}\left(X^{g}\right) e\left(\left(x Q+i y R^{g}\right)[X]\right),
$$

where $v(\Lambda)=\int_{\mathcal{V} / \Lambda} d x$ and

$$
c(h, l)_{\gamma}= \begin{cases}\delta_{h, a l} e(a b Q(h, l)) & \text { if } c=0 \\ |\operatorname{det} Q|^{1 / 2} v(\Lambda)^{-1}|c|^{-n / 2} \sum_{r \in \Lambda / c \Lambda} e\left(\frac{a Q[h+r]-2 Q(l, h+r)+d Q[l]}{c}\right) & \text { if } c \neq 0 .\end{cases}
$$

We remark that these expressions from $[8,21]$ are not accurate due to a certain confusion between the elements $r_{0}(g, Q)$ and $r(g, Q)$ in these references.

\section{The Main Result}

Let $N$ is a natural number such that $N_{1} \mid N$.

Definition 3.1. The regularized lifting of $f \in W M F_{k,-\lambda}^{+}(\Gamma(N))$ associated to $\Lambda$ and $V$ is given by $F^{r e g}(g, V, \Lambda ; f)={ }^{t}\left(F_{1-k}^{r e g}, \cdots, F_{k-1}^{r e g}\right)$, where for $|\alpha| \leq k-1$ the component $F_{\alpha}^{r e g}(g, V, \Lambda ; f)$ is defined by

$$
F_{\alpha}^{r e g}(g, V, \Lambda ; f):=\int_{\Gamma(N) \backslash \mathfrak{H}}^{r e g} \theta_{k, \alpha}(z, V, g, \Lambda) \overline{f(z)} y^{k-2} d x d y .
$$

We remark that this lift is the complex conjugate of the lift in [2], in the sense that it is anti-linear rather than linear as a function of the lifted modular form $f$.

Theorem 3.2. The lifted forms $F^{r e g}(g(\mu, \nu) ; f)$ on $\mathfrak{H}^{1}$ are weak Maass forms for $K$ of weight $2 k-2$ and eigenvalue $\left(k^{2}-2 k\right) / 4+\lambda$ for the group $G(\Lambda):=\{\gamma \in$ $\left.\mathbf{G} \mid \Lambda^{\gamma}=\Lambda\right\}$ with singularities on

$$
\bigcup_{\gamma \in \Gamma(N) \backslash S L_{2}(\mathbb{Z})} \bigcup_{\substack{X \in \Lambda^{*} \\-n_{\gamma} \leq \frac{N Q[X]}{2} \leq-1}}\left\{\mu+\nu \mathbf{k} \in \mathfrak{H}^{1} \mid\left(Q+R^{g(\mu, \nu)}\right)[X]=0\right\}
$$

where $n_{\gamma}$ is the index coming from the Fourier expansion (1) of $f$. 
In this section we prove Theorem 3.2. To show the convergence of $F_{\alpha}^{r e g}(g, V, \Lambda ; f)$, we need the convergence of the function

$$
\int_{1}^{t} \int_{-1 / 2}^{1 / 2} \sum_{\gamma \in \Gamma(N) \backslash S L_{2}(\mathbb{Z})}\left(\left.\theta_{k, \alpha}\right|_{k} \gamma\right)(z) \overline{\left(\left.f\right|_{k} \gamma\right)(z)} y^{k-2-w} d x d y
$$

as $t \rightarrow \infty$, for $\operatorname{Re}(w) \gg 0$. Since $\sum_{\gamma \in \Gamma(N) \backslash S L_{2}(\mathbb{Z})}\left(\left.\theta_{k, \alpha}\right|_{k} \gamma\right)(z) \overline{\left(\left.f\right|_{k} \gamma\right)(z)} y^{k}$ is $S L_{2}(\mathbb{Z})$-invariant, we may write $\sum_{\gamma \in \Gamma(N) \backslash S L_{2}(\mathbb{Z})}\left(\left.\theta_{k, \alpha}\right|_{k} \gamma\right)(z) \overline{\left(\left.f\right|_{k} \gamma(z)\right.} y^{k}=\sum_{n \in \mathbb{Z}}$ $a(n, y, V, g, \Lambda) e^{2 \pi i n x}$. Then

$$
\begin{aligned}
& \int_{1}^{t} \int_{-1 / 2}^{1 / 2} \sum_{\gamma \in \Gamma(N) \backslash S L_{2}(\mathbb{Z})}\left(\left.\theta_{k, \alpha}\right|_{k} \gamma\right)(z) \overline{\left(\left.f\right|_{k} \gamma\right)(z)} y^{k-2-w} d x d y \\
= & \int_{1}^{t} \int_{-1 / 2}^{1 / 2} \sum_{n \in \mathbb{Z}} a(n, y, V, g, \Lambda) e^{2 \pi i n x} y^{-2-w} d x d y=\int_{1}^{t} a(0, y, V, g, \Lambda) y^{-2-w} d y .
\end{aligned}
$$

Hence to ensure the convergence of the regularized theta lifting, we need to know the growth condition of the 'constant term' of $\sum_{\gamma \in \Gamma(N) \backslash S L_{2}(\mathbb{Z})}\left(\left.\theta_{k, \alpha}\right|_{k} \gamma\right)(z) \overline{\left(\left.f\right|_{k} \gamma\right)(z)}$. Let $\left(\left.f\right|_{k} \gamma^{-1}\right)(z)=\sum_{n \in \mathbb{Z}} b_{\gamma}(n, y, s) e^{2 \pi i n x / N}$ for $\gamma=\left(\begin{array}{ll}a & b \\ c & d\end{array}\right) \in S L_{2}(\mathbb{Z})$. Then $\left(\left.\theta_{k, \alpha}\right|_{k} \gamma\right)(z, V, g, \Lambda) \overline{\left.f\right|_{k} \gamma(z)}$ is equal to

$$
i^{-s g n c} y^{\frac{1}{2}} \sum_{X \in \Lambda^{*}} \sum_{n \in \mathbb{Z}} c(-V, X)_{\gamma} \eta_{k, \alpha}\left(X^{g}\right) \overline{b_{\gamma^{-1}}(n, y, s)} e^{-\pi y R^{g}[X]} e^{\pi i x Q[X]} e^{-2 \pi i n x / N} .
$$

Since $\sum_{\gamma \in \Gamma(N) \backslash S L_{2}(\mathbb{Z})}\left(\left.\theta_{k, \alpha}\right|_{k} \gamma\right)(z, V, g, \Lambda) \overline{\left(\left.f\right|_{k} \gamma\right)(z)} y^{k}$ is $S L_{2}(\mathbb{Z})$-invariant and has the Fourier expansion of the form $\sum_{n \in \mathbb{Z}} a(n, y, V, g, \Lambda, s) e^{2 \pi i n x}$, summing all the expansions

$\left(\left.\theta_{k, \alpha}\right|_{k} \gamma\right)(z, V, g, \Lambda) \overline{\left(\left.f\right|_{k} \gamma\right)(z)} y^{k}$ over $\Gamma(N) \backslash S L_{2}(\mathbb{Z})$ we have only the terms involving $e^{2 \pi i n x}$ for $n$ integral, although each series $\left(\left.\theta_{k, \alpha}\right|_{k} \gamma\right)(z, V, g, \Lambda) \overline{\left(\left.f\right|_{k} \gamma\right)(z)} y^{k}$ has the terms involving $e^{2 \pi i n x / N}$ in the expansion. Consequently we need to consider the convergence of the following integral

$$
\begin{aligned}
& \int_{1}^{t} \sum_{\gamma \in \Gamma(N) \backslash S L_{2}(\mathbb{Z})} i^{-s g n c} \\
& \sum_{X \in \Lambda^{*}} c(-V, X)_{\gamma} \eta_{k, \alpha}\left(X^{g}\right) \overline{b_{\gamma^{-1}}\left(\frac{N Q[X]}{2}, y, s\right)} e^{-\pi y R^{g}[X]} y^{k-\frac{3}{2}-w} d y
\end{aligned}
$$

as $t \rightarrow \infty$ and therefore it has a finite limit as $t \rightarrow \infty$ if $\operatorname{Re}(w) \gg 0$. 


\subsection{Convergence Region}

In this subsection we complete the proof for the convergence of the regularized theta lifting and describe the convergence region of $F^{r e g}(g(\mu, \nu) ; f)$. Let $g(\mu, \nu)$ be a point in the complement of

$$
\bigcup_{\gamma \in \Gamma(N) \backslash S L_{2}(\mathbb{Z})} \bigcup_{\substack{X \in \Lambda^{*} \\-n_{\gamma} \leq N Q[X]} \leq-1}^{2}\left\{g=g(\mu, \nu) \mid\left(Q+R^{g}\right)[X]=0\right\} .
$$

Inputting the Fourier expansion of $\left(\left.f\right|_{k} \gamma\right)(z)$ into the expression of $F_{\alpha}^{r e g}(g(\mu, \nu) ; f)$ above, the estimates $a_{\gamma^{-1}}(n, s)=O\left(e^{\sqrt{n}}\right)$ and $W_{\nu, \mu}(y) \sim e^{-y / 2} y^{\nu}$ as $|y| \rightarrow \infty$ give the convergence for the part concerning $W_{ \pm \frac{k}{2}, s-\frac{1}{2}}(2 \pi|Q[X]| y)$. For the constant term we have the integral of the form $\int_{1}^{t} y^{\frac{k}{2}-\frac{1}{2}-s-w} d y$, therefore it converges for $\operatorname{Re}(w) \gg 0$, as $t \rightarrow \infty$. It remains to consider the part concerning $W_{\frac{k}{2}, s-\frac{1}{2}}(-2 \pi|Q[X]| y)$. It is a fact that for any $C \geq 0$ and any compact subset $U \subset \mathfrak{H}^{1}$ the set

$$
\left\{\lambda \in \Lambda^{*} \mid Q[\lambda]=m, \exists g=g(\mu, \nu) \in U \text { with }\left(Q+R^{g}\right)[\lambda] \leq C\right\}
$$

is finite. By assumption, there exists $\varepsilon>0$ such that $\left(Q+R^{g}\right)[\lambda]>\varepsilon$ for all $\lambda \in \Lambda^{*}$ with $Q[\lambda]=m$ for a fixed $g=g(\mu, \nu)$. It follows that

$$
\begin{aligned}
& \sum_{\substack{X \in \Lambda^{*} \\
Q[X]=m<0}}\left|c(-V, X)_{\gamma} \eta_{k, \alpha}\left(X^{g}\right) W_{\frac{k}{2}, \bar{s}-\frac{1}{2}}(2 \pi m y) e^{-\pi y R^{g}[X]} y^{\frac{1}{2} k-\frac{3}{2}-w}\right| \\
\ll & \sum_{\substack{X \in \Lambda^{*} \\
Q[X]=m<0}}\left|\eta_{k, \alpha}\left(X^{g}\right)\right| e^{\pi(-m+Q[X]) y} e^{-\pi y\left(Q+R^{g}\right)[X]} y^{k-\frac{3}{2}-R e(w)} \\
\ll & e^{-\frac{\pi \varepsilon y}{2}} y^{k-\frac{3}{2}-R e(w)} \sum_{\substack{X \in \Lambda^{*} \\
Q[X]=m<0}}\left|\eta_{k, \alpha}\left(X^{g}\right)\right| e^{-\pi\left(m+R^{g}[X]\right) / 2},
\end{aligned}
$$

which yields the uniform convergence for $y \in[1, \infty)$. This implies the desired convergence assertion.

\subsection{Modularity}

In this subsection we show that $F^{r e g}(g(\mu, \nu) ; f)$ satisfies the modularity as in Definition 2.2. We define an involution $\iota_{n}$ on the space of modular forms for $K$ of weight $n$ by $\iota_{n} F^{1}(z)=\rho_{n}\left(\begin{array}{ll}0 & 1 \\ 1 & 0\end{array}\right) F^{1}(-\bar{z})$. Note that each $G\left(\Lambda_{i}\right)$ is a congruence subgroup of $S L_{2}\left(\mathcal{O}_{K}\right)_{i}$ (see [10]). The basic properties of the lifted form $F^{r e g}(g(\mu, \nu) ; f)$ are as follows.

Theorem 3.3. The lifted form $F^{r e g}(g(\mu, \nu) ; f)$ satisfies the following properties.

(1) For $\kappa$ in $S U(2, \mathbb{C})$ and $\gamma$ in $G(\Lambda), F^{r e g}(\gamma g \kappa, V)={ }^{t} \rho_{2 k-2}(\kappa) F^{r e g}\left(g, V^{\gamma}\right)$. 
(2) $F^{r e g}(g, V, \Lambda)=(-1)^{k-1} \rho_{2 k-2}\left(\begin{array}{cc}0 & -1 \\ 1 & 0\end{array}\right) F^{r e g}(\bar{g}, \bar{V}, \bar{\Lambda})$.

(3) $F^{r e g}\left({ }^{t} g^{-1}, V, \Lambda\right)=(-1)^{k-1} F^{r e g}(\bar{g}, \operatorname{adj}(V), \operatorname{adj}(\Lambda))$.

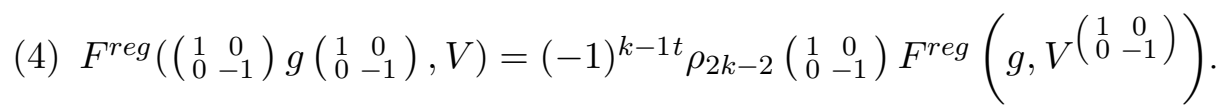

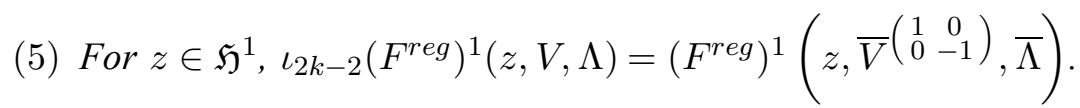

This is a generalization of Theorem 3.3 in [10]. The proof is almost identical to the proof of Theorem 3.3 of [10], hence will not be repeated here. We note that in particular Proposition 2.3 and Theorem 3.3 (1) ensures that the function $\left(F^{r e g}(g(\mu, \nu) ; f)\right)^{1}$ on $\mathfrak{H}^{1}$ corresponding to $F^{\text {reg }}(g(\mu, \nu) ; f)$ is modular of weight $2 k-2$.

\subsection{Casimir operators}

In this subsection we describe the behavior of $F^{r e g}(g(\mu, \nu) ; f)$ under the Casimir operators and prove that $F_{\alpha}^{r e g}(g(\mu, \nu) ; f)$ is compatible with the Maass differential operators and the Laplacian. This was already illustrated in the paper of Friedberg $[10,12]$ when $f$ is a Maass cusp form. From now on we generalize it to weak Maass forms. We only consider the behavior under the Casimir operators, but the same result holds for Maass operators. Recall that $\Delta_{k}=L_{k+2} R_{k}-k=R_{k-2} L_{k}$, where $R_{k}$ and $L_{k}$ are Maass operators given by $R_{k}=2 i \frac{\partial}{\partial z}+\frac{k}{y}$ and $L_{k}=2 i y^{2} \frac{\partial}{\partial \bar{z}}$. Let $\mathcal{F}_{u}=\left\{z=x+i y \in \mathfrak{H}|| z|\geq 1| x \mid, \leq \frac{1}{2}, y \leq u\right\}$ denote the truncated fundamental domain for $\Gamma(1)$. We recall the following

Lemma 3.4. ([5, Lemma 4.2]). Let $f$ and $g$ be smooth functions such that $\left.f\right|_{k+2} \gamma=f$ and $\left.g\right|_{k} \gamma=g$. Then for all $\gamma \in \Gamma(N)$

$$
\begin{aligned}
& \int_{-\frac{1}{2}}^{\frac{1}{2}}\left[\sum_{\gamma} y^{k} \overline{\left(\left.f\right|_{k+2} \gamma\right)(z)}\left(\left.g\right|_{k} \gamma\right)(z)\right]_{y=u} d x \\
= & \left.\int_{\mathcal{F}_{u}} y^{k-2} \sum_{\gamma} \overline{L_{k+2}\left(\left.f\right|_{k+2} \gamma\right)(z)} g\right|_{k} \gamma(z) d x d y \\
& -\int_{\mathcal{F}_{u}} y^{k} \sum_{\gamma} \overline{\left(\left.f\right|_{k+2} \gamma\right)(z)} R_{k}\left(\left.g\right|_{k} \gamma\right)(z) d x d y .
\end{aligned}
$$

Recall that $\left(\left.\theta_{k, \alpha}\right|_{k} \gamma\right)(z, V, g, \Lambda)=\theta_{k, \alpha}(z, V, g, \Lambda)$ for all $\gamma \in \Gamma(N)$ and $N_{1} \mid N$. Hence we can apply Lemma 3.4 to a pair of $\theta_{k, \alpha}(z, V, g, \Lambda)$ and $f(z)$. Then the key fact is the following theorem in [10] describing the behavior of the theta kernel $\theta_{k, \alpha}(z, V, g, \Lambda)$ under Casimir operators corresponding to $S L_{2}(\mathbb{R})$ and $S L_{2}(\mathbb{C})$.

Theorem 3.5. ([10, Theorem 3.4]). $\Psi \theta_{k, \alpha}(z, g)=\tilde{\Psi} \theta_{k, \alpha}(z, g)=\left(-\Delta_{k}+\frac{k^{2}-2 k}{4}\right)$ $\theta_{k, \alpha}(z, g)$. 
For simplicity, we write $F_{\alpha}^{r e g}(g):=F_{\alpha}^{r e g}(g, V, \Lambda ; f)$. We also use the notation $\left\langle\theta_{k, \alpha}, f\right\rangle^{r e g}$ for $F_{\alpha}^{r e g}(g, V, \Lambda ; f)$. By Theorem 3.5

$$
\begin{aligned}
\left(\Psi F_{\alpha}^{r e g}\right)(g) & =\left(\tilde{\Psi} F_{\alpha}^{r e g}\right)(g)=\left\langle\Psi \theta_{k, \alpha}(z, g), f(z)\right\rangle^{r e g} \\
& =\left\langle\left(-\Delta_{k}+\frac{k^{2}-2 k}{4}\right) \theta_{k, \alpha}(z, g), f(z)\right\rangle^{r e g} .
\end{aligned}
$$

Applying Lemma 3.4 twice and following the argument of [5] we get

$$
\begin{aligned}
& \left\langle\left(-\Delta_{k}+\frac{k^{2}-2 k}{4}\right) \theta_{k, \alpha}(z, g), f(z)\right\rangle^{r e g} \\
= & -\lim _{u \rightarrow \infty} \int_{\mathcal{F}_{u}} \sum_{\gamma \in \Gamma(N) \backslash S L_{2}(\mathbb{Z})}\left(\left.\theta\right|_{k} \gamma\right)(z) \frac{\left(\Delta_{k}-\frac{k^{2}-2 k}{4}\right)\left(\left.f\right|_{k} \gamma\right)(z)}{4 k-2} d x d y \\
= & -\left\langle\theta_{k, \alpha}(z, g),\left(\Delta_{k}-\frac{k^{2}-2 k}{4}\right) f(z)\right\rangle^{r e g} \\
= & -\left\langle\theta_{k, \alpha}(z, g),\left(-\lambda-\frac{k^{2}-2 k}{4}\right) f(z)\right\rangle^{r e g} \\
= & \left(\frac{k^{2}-2 k}{4}+\lambda\right) F_{\alpha}^{r e g}(g),
\end{aligned}
$$

and in conclusion

$$
\left(\Psi F_{\alpha}^{r e g}\right)(g)=\left(\tilde{\Psi} F_{\alpha}^{r e g}\right)(g)=\left(\frac{k^{2}-2 k}{4}+\lambda\right) F_{\alpha}^{r e g}(g) .
$$

4. Singularity Type And the Fourier Expansion of $F_{\alpha}^{\text {reg }}$

In this section we compute the singularity type of $F_{\alpha}^{r e g}(g(\mu, \nu) ; f)$ and its Fourier expansion.

\subsection{Singularity type}

First we consider the singularity type. Let $U \subset \mathfrak{H}^{1}$ be an open subset and $f, g$ functions on a dense open subset of $U$. Then we write $f \approx g$ if $f-g$ can be continued to a real analytic function on $U$. In this case we say that $f$ has a singularity of type $g$. For a subset $U$ of $\mathfrak{H}^{1}$ with compact closure, we define

$$
\mathcal{S}(m, U):=\left\{\lambda \in \Lambda^{*} \mid \frac{N Q[\lambda]}{2}=m, \exists \mu+\nu \mathbf{k} \in U,\left(Q+R^{g(\mu, \nu)}\right)[\lambda]=0\right\} .
$$

Note that $\mathcal{S}(m, U)$ is a finite set. 
Theorem 4.1. Let $U \subset \mathfrak{H}^{1}$ be an open subset with compact closure. On $U$ the function $F_{\alpha}^{r e g}(g(\mu, \nu) ; f)$ has a singularity of type

$$
\begin{aligned}
& (-1)^{\frac{k}{2}} \sum_{n=0}^{k-1}\left\{1+\frac{\left\{\left(\bar{s}-\frac{1}{2}\right)^{2}-\left(\frac{k}{2}-\frac{1}{2}\right)^{2}\right\} \cdots\left\{\left(\bar{s}-\frac{1}{2}\right)^{2}-\left(\frac{k}{2}-n+\frac{1}{2}\right)^{2}\right\}}{n !}\right\} \\
& \quad \times \sum_{\gamma \in \Gamma(N) \backslash S L_{2}(\mathbb{Z})} i^{-\operatorname{sgn} c} \sum_{m<0} \frac{\overline{\tilde{a}}_{\gamma^{-1}\left(\frac{m}{2}, s\right)}^{(2 \pi m)^{n}}}{\sum_{X \in \mathcal{S}(m, U)} c(-V, X)_{\gamma} \eta_{k, \alpha}\left(X^{g(\mu, \nu)}\right)} \\
& \quad \times \Gamma\left(k-n-\frac{3}{2}\right){\sqrt{\pi\left(m+R^{g(\mu, \nu)}[X]\right)}}^{2 n-2 k+3} \cdot
\end{aligned}
$$

Here $\overline{\tilde{a}_{\gamma^{-1}}\left(\frac{m}{2}, s\right)}$ are the Fourier coefficients of $\left.f\right|_{k} \gamma, c(-V, X)_{\gamma}$ are the factors appearing in the Fourier expansion of $\left.\theta_{k, \alpha}\right|_{k} \gamma$ and $\eta_{k, \alpha}\left(X^{g(\mu, \nu)}\right)$ is the spherical harmonic polynomials given in the definition of $\theta_{k, \alpha}$ (for the details, see section 2).

Again put the Fourier expansion of $\left(\left.f\right|_{k} \gamma\right)(z)$ inside as above. Then the part concerning $W_{ \pm \frac{k}{2}, s-\frac{1}{2}}(2 \pi|Q[\lambda]| y)$ and the constant term do not contribute to the singularity. In the remaining part concerning $W_{\frac{k}{2}, s-\frac{1}{2}}(-2 \pi|Q[\lambda]| y)$, the integrals become singular if $\left(Q+R^{g}\right)[\lambda]=0$, hence we have the singularity type

$$
\begin{aligned}
& \sum_{\gamma \in \Gamma(N) \backslash S L_{2}(\mathbb{Z})} i^{-s g n c} \sum_{m<0} \overline{\tilde{a}_{\gamma^{-1}}\left(\frac{m}{2}, s\right)} \sum_{X \in \mathcal{S}(m, U)}|2 \pi m|^{-\frac{k}{2}} c(-V, X)_{\gamma} \eta_{k, \alpha}\left(X^{g}\right) \\
& \times \int_{1}^{\infty} W_{\frac{k}{2}, \bar{s}-\frac{1}{2}}(2 \pi m y) e^{-\pi y R^{g}[X]} y^{\frac{k}{2}-\frac{3}{2}} d y
\end{aligned}
$$

Note that the integral is evaluated near the singular point but not at it. It is known that $W_{p, q}(z)$ has the following asymptotic expansion (see [22, p.345])

$$
\begin{aligned}
& W_{p, q}(z) \\
= & e^{-\frac{z}{2}} z^{p}\left\{1+\sum_{L=1}^{n} \frac{\left\{q^{2}-\left(p-\frac{1}{2}\right)^{2}\right\}\left\{q^{2}-\left(p-\frac{3}{2}\right)^{2}\right\} \cdots\left\{q^{2}-\left(p-L+\frac{1}{2}\right)^{2}\right\}}{L ! z^{L}}+O\left(\frac{1}{z^{n+1}}\right)\right\}
\end{aligned}
$$

as $|z| \rightarrow \infty,|\arg z|<\frac{3}{2} \pi$. We apply this to $W_{\frac{k}{2}, \bar{s}-\frac{1}{2}}(2 \pi m y)$ with $L=k-1$. This makes the integral associated to the error terms converge, i.e.

$$
\begin{aligned}
& \int_{1}^{\infty} e^{-\pi m y}|2 \pi m y|^{\frac{k}{2}} \cdot C|2 \pi m y|^{-L-1} e^{-\pi y R^{g}[X]} y^{\frac{k}{2}-\frac{3}{2}} d y \\
= & C|2 \pi m|^{\frac{k}{2}-L-1}{\sqrt{\pi\left(m+R^{g}[X]\right)}}^{2 L-2 k+3} \int_{\pi\left(m+R^{g}[X]\right)}^{\infty} e^{-y} y^{k-L-\frac{5}{2}} d y .
\end{aligned}
$$


The latter integral is uniformly bounded since $k-L-\frac{5}{2} \notin \mathbb{Z}$ and $2 L-2 k+3>0$, so it converges to zero as $m+R^{g}[X] \rightarrow 0$. Hence we may use this asymptotic formula and get

$$
\begin{aligned}
& \int_{1}^{\infty} W_{\frac{k}{2}, \bar{s}-\frac{1}{2}}(2 \pi m y) e^{-\pi y R^{g}[X]} y^{\frac{k}{2}-\frac{3}{2}} d y \\
\sim & \sum_{n=0}^{k-1}\left\{1+\frac{\left\{\left(\bar{s}-\frac{1}{2}\right)^{2}-\left(\frac{k}{2}-\frac{1}{2}\right)^{2}\right\} \cdots\left\{\left(\bar{s}-\frac{1}{2}\right)^{2}-\left(\frac{k}{2}-n+\frac{1}{2}\right)^{2}\right\}}{n !(2 \pi m)^{n}}\right\}(2 \pi m)^{\frac{k}{2}} \\
& \times{\sqrt{\pi\left(m+R^{g}[X]\right)^{2 n-2 k+3}} \Gamma\left(k-n-\frac{3}{2}\right),}^{2 n}(k)
\end{aligned}
$$

which completes the proof.

\subsection{Fourier expansion}

In this subsection we compute the Fourier expansion of $F_{\alpha}^{r e g}(g(\mu, \nu) ; f)$. Let $\Lambda$ be one of the $\Lambda_{i}$ 's above and $J$ the corresponding $J_{i}$. Write $V \in \Lambda^{*}$ as $V=\left(\begin{array}{l}v_{1} \\ v_{2} \\ v_{4}\end{array}\right)$, and for $X=\left(\frac{m}{r} p\right) \in \mathcal{P}$ put $X-V=\left(\begin{array}{cc}m_{1} & r_{2} \\ \bar{r}_{2} & p_{4}\end{array}\right)$. Take a set of coset representatives $\mathcal{R}$ for $\left(\begin{array}{ll}1 & \mathbb{Z} \\ 0 & 1\end{array}\right) \backslash S L_{2}(\mathbb{Z}) / \Gamma(N)$ such that every $\rho=\left(\begin{array}{cc}\rho_{1} & \rho_{2} \\ \rho_{3} & \rho_{4}\end{array}\right) \in \mathcal{R}$ has $\rho_{3} \neq 0$. Let $C_{\alpha}=\frac{(k-1) ! N i^{k-\alpha}}{\left(\mathbf{N}(I)^{2} D\right)^{1 / 2} \pi^{k} 2^{2 k-2}}$. For a given $f \in W M F_{k,-\lambda}^{+}(\Gamma(N))$ we have $\tilde{m} \in \mathbb{Z}$ such that $\left.f\right|_{k} \gamma^{-1}=O\left(e^{2 \pi \tilde{m} y}\right)$ for all $\gamma \in\left(\begin{array}{c}1 \\ 0\end{array}\right) \backslash S L_{2}(\mathbb{Z}) / \Gamma(N)$. Let $\hat{m}$ be $\left(2 \tilde{m} P^{2}\right)^{1 / 2}$ if $\tilde{m} \geq 0$ and 0 otherwise. Since $F_{\alpha}^{r e g}(g, V, \Lambda ; f)=(-1)^{\alpha} F_{-\alpha}^{r e g}(\bar{g}, \bar{V}, \bar{\Lambda} ; f)$ for $\alpha \leq 0$, it is enough to compute the Fourier expansion in the case $\alpha \geq 0$. To state the result, we define

$$
\begin{aligned}
p^{\prime}(\nu, s, \rho)= & \nu^{2-2 \bar{s}} \pi^{-\frac{k}{2}+1-\bar{s}} q(k, s) \sum_{\substack{t=1 \\
t \rho_{3} \equiv-P v_{1}(\bmod M P)}}\left(P^{-1} t\right)^{1-2 \bar{s}} e\left(-2 P^{-1} t \rho_{4} v_{4}\right) \\
& \text { where } q(k, s)=\sum_{\substack{2 \beta+\gamma=k-1 \\
\beta, \gamma \geq 0}} \frac{(2 i)^{\gamma}}{\beta ! \gamma !} \Gamma\left(\bar{s}+\frac{k-1+\gamma}{2}\right), \\
\tau(\rho)= & \sum_{v^{\prime} \in \bar{J}^{-1} \mathcal{D}_{K}^{-1} /\left(-\rho_{3}\right) J^{v^{\prime \prime} \in J /\left(-\rho_{3}\right) J}} e\left(-2 \rho_{3}^{-1}\left(\rho_{4} \mathbf{N}\left(v^{\prime \prime}\right)\right.\right. \\
& \left.\left.+\rho_{1} \mathbf{N}\left(v^{\prime}\right)\right)+\operatorname{Tr}_{\mathbb{R}}^{\mathbb{C}}\left\{-2 \bar{v}^{\prime} v^{\prime \prime} \rho_{3}^{-1}\right\}\right), \\
p(\nu, w)= & \frac{(k-1) ! P^{2 w-1} N i}{2^{k-1}\left(\mathbf{N}(I)^{2} D\right)^{1 / 2}} \sum_{\rho \in \mathcal{R}} \rho_{3}^{-1} \tau(\rho) e\left(2 \rho_{4} \rho_{2} \mathbf{N}\left(v_{2}\right)\right) \\
& \times\left(\overline{\tilde{a}_{\rho}(0, s)} p^{\prime}(\nu, s+w, \rho)+\overline{a_{\rho}(0, s)} p^{\prime}(\nu, 1-s+w, \rho)\right) .
\end{aligned}
$$

We also let 


$$
\begin{aligned}
\tau\left(\rho, r, v_{2}\right)= & \rho_{3}^{-1} e\left(-2 \rho_{3}^{-1}\left(\rho_{1} \mathbf{N}(r)+\operatorname{Tr}_{\mathbb{R}}^{\mathbb{C}}\left(v_{2} \bar{r}\right)+\rho_{4} \mathbf{N}\left(v_{2}\right)\right)\right) \\
& \times \sum_{j \in J / \rho_{3} J} e\left(-2 \rho_{3}^{-1}\left(\operatorname{Tr}_{\mathbb{R}}^{\mathbb{C}}\left(\bar{j}\left(r+\rho_{4} v_{2}\right)\right)+\rho_{4} \mathbf{N}(j)\right)\right),
\end{aligned}
$$

$$
\begin{aligned}
& q_{\alpha, \beta, \gamma}(y, \lambda) \\
:= & \sum_{m=0}^{\beta} \sum_{e=0}^{\beta+\gamma-m}(-1)^{\gamma+e}\left(\begin{array}{c}
\beta+\gamma-m \\
e
\end{array}\right)[(\beta-m) !(\alpha+m) ! m ! \gamma !]^{-1} 2^{\gamma} \\
& \times\left(\frac{k}{2}-s\right)_{\beta+\gamma-m-e} y^{2 m+e+\alpha+1} K_{2 s-1-e}(2 y),
\end{aligned}
$$

where $(a)_{n}=a(a-1) \cdots(a-n+1)$ with $(a)_{0}=1$. Let $q_{\alpha}(y, \lambda)=\sum_{\beta, \gamma} q_{\alpha, \beta, \gamma}(y, \lambda)$, where the sum is over nonnegative integers $\beta$ and $\gamma$ such that $2 \beta+\gamma=k-\alpha-1$ and $\alpha+\beta \geq 0$.

Theorem 4.2. Let $\alpha \geq 0$. Let $f \in W M F_{k,-\lambda}^{+}(\Gamma(N))$ with a Fourier expansion of the form (1). Let $V=\left(\frac{v_{1}}{v_{2}} v_{4}\right) \in \Lambda^{*}$. Then for $\nu>\hat{m}$ the lifted form $F_{\alpha}^{r e g}(g(\mu, \nu) ; f)$ has the Fourier expansion given by the constant term of the Laurent expansion at $w=0$ of the analytic continuation of

$$
\begin{gathered}
C_{\alpha} \sum_{\rho \in \mathcal{R}} \sum_{\substack{t=1 \\
t \rho_{3} \equiv-P v_{1}(\bmod M P)}}^{\infty} t^{-1} \sum_{r \in \bar{J}^{-1} \mathcal{D}_{K}^{-1}-0}|r|^{-k}(r /|r|)^{\alpha} \overline{a_{\rho}(N \mathbf{N}(r), s)} \\
\times \tau\left(\rho, r, v_{2}\right) q_{\alpha}\left(2 \pi P^{-1} t \nu|r|, \lambda\right) e\left(2 P^{-1} t \operatorname{tr}(\bar{r} \mu)-\rho_{4} v_{4}\right)+\delta_{\alpha, 0} p(\nu, w) \\
+(k-1) !\left[\left(\frac{k-1+\alpha}{2}\right) !(4 \pi)^{(k-1-\alpha) / 2}\right]^{-1} \\
\quad \delta_{v_{1}(\bmod M), 0} \nu\left\langle\phi_{\alpha,(k-1-\alpha) / 2}\left(z, 0, v_{2}\right), f\right\rangle^{r e g},
\end{gathered}
$$

where $\tau\left(\rho, r, v_{2}\right)$ and $q_{\alpha}\left(2 \pi P^{-1} t \nu|r|, \lambda\right)$ are the functions defined in (5) and (6).

In [10, Theorem 4.1] the Fourier expansion of $F_{\alpha}^{r e g}(g(\mu, \nu) ; f)$ has been derived in the case when $f$ is a Maass cusp form. Compared to this, our result coincide with [10, Theorem 4.1] in the first two parts and contains extra summands whose main terms are $\nu^{2-2 \bar{s}}$ and $\nu^{2 \bar{s}}$. Proof. We proceed as in the proof of Theorem 4.1 in [10] together with the convergence analysis. Let us first consider the $\alpha \geq 0$ case. Note that $\left\langle\theta_{k, \alpha}, f\right\rangle^{r e g}=\left\langle\left.\theta_{k, \alpha}\right|_{k} \rho^{-1},\left.f\right|_{k} \rho^{-1}\right\rangle^{r e g}$. This is because $\left.\left.\sum_{\gamma \in \Gamma(N) \backslash S L_{2}(\mathbb{Z})} \theta_{k, \alpha}\right|_{k} \gamma \bar{f}\right|_{k} \gamma y^{k}$ is $S L_{2}(\mathbb{Z})$-invariant. Using the Rankin-Selberg method we unfold the integral. First $\phi_{\alpha, \beta}(z, 0, v) \in G\{\Gamma(N), \alpha+2 \beta+1,-\beta(\alpha+\beta)\}$ for $v \in \bar{J}^{-1} \mathcal{D}_{K}^{-1}$, when we consider also the behavior under Maass operators, where $D \mathbf{N}(I) \mid N$. We compute the lifting 
using Proposition 4.6 of [10] to obtain that $\left\langle\theta_{k, \alpha}, f\right\rangle^{r e g}$ is equal to

$$
\begin{aligned}
& \int_{\Gamma(N) \backslash \mathfrak{H}}^{r e g}\left[\sum_{\beta, \gamma} \frac{(k-1) ! i^{\gamma} \nu^{\gamma+1}}{(\alpha+\beta) ! \gamma ! 2^{\beta}(2 \pi)^{\beta} P} \times\left\{\delta_{v_{1}(\bmod M), 0} \delta_{\gamma, 0} \phi_{\alpha, \beta}\left(z, 0, v_{2}\right)\right.\right. \\
& \quad+\sum_{\rho \in \mathcal{R}} \sum_{\substack{t=1 \\
t \rho_{3} \equiv-P v_{1}(\bmod M P)}}\left(P^{-1} t\right)^{\gamma} \sum_{\sigma \in \Gamma \Gamma_{\infty} \backslash \Gamma(N)} \\
& \quad\left[\left(\left.\phi_{\alpha, \beta}\left(z, P^{-1} t \mu \rho_{4}, v_{2}-P^{-1} t \mu \rho_{3}\right)\right|_{\alpha+2 \beta+1} \rho^{-1}\right.\right. \\
& \left.\left.\left.\left.\quad \times y^{-\gamma} e\left(i P^{-2} t^{2} \nu^{2} y^{-1}-P^{-1} t\left(\operatorname{Tr}_{\mathbb{R}}^{\mathbb{C}}\left(v_{2} \bar{\mu} \rho_{4}\right)+2 \rho_{4} v_{4}\right)\right)\right)\left.\right|_{k} \sigma\right](z)\right\}\right] \overline{\left.f\right|_{k} \rho^{-1}} y^{k} \frac{d x d y}{y^{2}} .
\end{aligned}
$$

The constant term $\delta_{v_{1}(\bmod M), 0} \delta_{\gamma, 0} \phi_{\alpha, \beta}\left(z, 0, v_{2}\right)$ in the expansion of $\theta_{k, \alpha}$ is a modular form for $\Gamma(N)$ of weight $k=\alpha+2 \beta+1$ if this is nontrivial, i.e., $\gamma=0$, so as above we have

$$
\begin{aligned}
& \left\langle\delta_{v_{1}(\bmod M), 0} \phi_{\alpha,(k-1-\alpha) / 2}\left(z, 0, v_{2}\right), f\right\rangle^{r e g} \\
= & \left\langle\left.\delta_{v_{1}(\bmod M), 0} \phi_{\alpha,(k-1-\alpha) / 2}\left(z, 0, v_{2}\right)\right|_{k} \rho^{-1},\left.f\right|_{k} \rho^{-1}\right\rangle^{r e g},
\end{aligned}
$$

which gives the constant term in the expansion of $F_{\alpha}^{r e g}(g(\mu, \nu) ; f)$.

We begin with analyzing this constant term. It converges because $\phi_{\alpha, \beta}$ contains only Fourier coefficients with positive indices, so that only decreasing Whittaker functions and exponentials survive after integration with respect to $x$ (up to the constant Fourier coefficients, which are dealt with using the extra $y^{-w}$ ). For evaluating it we take $v$ with $v_{1} \equiv 0(\bmod M)$, and obtain that

$$
\begin{aligned}
& \left\langle\phi_{\alpha, \frac{k-1-\alpha}{2}}\left(z, 0, v_{2}\right), f\right\rangle^{r e g} \\
= & \int_{S L_{2}(\mathbb{Z}) \backslash \mathfrak{H}}^{r e g} \sum_{\gamma \in \Gamma(N) \backslash S L_{2}(\mathbb{Z})}\left(\left.\phi_{\alpha, \frac{k-1-\alpha}{2}}\right|_{k} \gamma\right)\left(z, 0, v_{2}\right) \overline{\left.f\right|_{k} \gamma(z)} y^{k} \frac{d x d y}{y^{2}} .
\end{aligned}
$$

It suffices to consider the integration over the subregion satisfying the condition $y>1$ for convergence check. So we consider the integral taken over that region. Next we separate the integral into two parts

$$
\begin{aligned}
& \left\langle\phi_{\alpha, \frac{k-1-\alpha}{2}}\left(z, 0, v_{2}\right), f\right\rangle^{r e g} \\
= & \int_{1}^{\infty} \int_{0}^{1} \sum_{\substack{\gamma \in \Gamma(N) \backslash S L_{2}(\mathbb{Z}) \\
c=0}}\left(\left.\phi_{\alpha, \frac{k-1-\alpha}{2}}\right|_{k} \gamma\right)\left(z, 0, v_{2}\right) \overline{\left.f\right|_{k} \gamma(z)} y^{k-w} \frac{d x d y}{y^{2}} \\
& +\int_{1}^{\infty} \int_{0}^{1} \sum_{\substack{\gamma \in \Gamma(N) \backslash S L_{2}(\mathbb{Z}) \\
c \neq 0}}\left\{(-1)^{\alpha+1} c^{k-2} \sum_{v^{\prime} \in \bar{J}^{-1} \mathcal{D}_{K}^{-1} / c J} \tau^{\prime}\left(\gamma, 0, v_{2}, v^{\prime}\right)\right. \\
& \left.\times \phi_{\alpha, \frac{k-1-\alpha}{2}}\left(c^{2} z, c b v_{2},-c^{-1}\left(a v_{2}-v^{\prime}\right)\right)\right\} \overline{\left.f\right|_{k} \gamma(z)} y^{k-w} \frac{d x d y}{y^{2}},
\end{aligned}
$$


where we have used the expression of $\left.\phi_{\alpha, \beta}\right|_{k} \gamma$ in [10, Proposition 4.7] in the second part. Here

$$
\begin{aligned}
& \tau^{\prime}\left(\gamma, u, v, v^{\prime}\right) \\
= & \left.\left(\mathbf{N}(I)^{2} D\right)^{-1 / 2} \sum_{v^{\prime \prime} \in J / c J} e\left(2 c^{-1}\left(c \mathbf{N}\left(v^{\prime \prime}\right)+d \mathbf{N}\left(v^{\prime}\right)\right)-\operatorname{Tr}_{\mathbb{R}}^{\mathbb{C}} \overline{v^{\prime}}\left(b v-d u-2 v^{\prime \prime} c^{-1}\right)\right)\right) .
\end{aligned}
$$

Note that in the first part, each $\left(\left.\phi_{\alpha, \frac{k-1-\alpha}{2}}\right|_{k} \gamma\right)\left(z, 0, v_{2}\right)$ are just translations by $m \in \mathbb{Z} / N \mathbb{Z}$. So the first part is equal to

$$
\int_{1}^{\infty} \int_{0}^{N} \phi_{\alpha, \frac{k-1-\alpha}{2}}\left(z, 0, v_{2}\right) \overline{f(z)} y^{k-w} \frac{d x d y}{y^{2}} .
$$

Then the term concerning $W_{\frac{k}{2}, s-\frac{1}{2}}\left(4 \pi \mathbf{N}\left(r-v_{2}\right) y\right)$ is of exponential decay and we can make the exponent of $y$ less than -1 for $\operatorname{Re}(w)$ sufficiently large, hence we conclude that the regularized integral converges.

Next we consider the second part. First input the Fourier expansion of $\phi_{\alpha, \frac{k-1-\alpha}{2}}$ and the Fourier expansion of $\left.f\right|_{k} \gamma(z)$ in the second part, and integrate it with respect to $x$. Then it suffices to show that the summation of each integration for each $r$ yields an absolutely convergent series using Lebesgue's dominated convergence theorem. Here the important summation is the sum over $r \in J$, because this is an infinite sum. But the growth conditions $a_{\gamma^{-1}}(n, s)=O\left(e^{C \sqrt{n}}\right)$ for some $C>0$ and $W_{\nu, \mu}(y) \sim$ $e^{-y / 2} y^{\nu}$ as $|y| \rightarrow \infty$ guarantee the convergence corresponding to $W_{\frac{k}{2}, s-\frac{1}{2}}$. It yields the convergence of the constant term of lifting.

Next we evaluate the 'non-constant terms'. Note that $\left(\left.\left(\left.f\right|_{k} \rho^{-1}\right)\right|_{k} \gamma\right)(z)=\left(\left.f\right|_{k} \rho^{-1}\right)(z)$ for all $\gamma \in \Gamma(N)$, because $\Gamma(N) \triangleleft S L_{2}(\mathbb{Z})$. Then we can replace the integral over a fundamental domain of $S L_{2}(\mathbb{Z})$ by an integral over a fundamental domain of $N \mathbb{Z}$, since the argument appearing in [2, Theorem 7.1] holds equally well here. We obtain

$$
\begin{aligned}
\int_{0}^{\infty} & \int_{0}^{N}\left[\sum_{\beta, \gamma} \frac{(k-1) ! i^{\gamma} \nu^{\gamma+1}}{(\alpha+\beta) ! \gamma ! 2^{\beta}(2 \pi)^{\beta} P}\right. \\
& \sum_{\rho \in \mathcal{R}} \sum_{\substack{t \rho_{3} \equiv-P v_{1}(\bmod M P)\\
}}\left(P^{-1} t\right)^{\gamma}\left(\phi _ { \alpha , \beta } \left(z, P^{-1} t \mu \rho_{4}, v_{2}-P^{-1} t\right.\right. \\
& \left.\times \mu \rho_{3}\right)\left.\right|_{\alpha+2 \beta+1} \rho^{-1} \cdot y^{-\gamma} e\left(i P^{-2} t^{2} \nu^{2} y^{-1}\right. \\
& \left.\left.\left.-P^{-1} t\left(\operatorname{Tr}_{\mathbb{R}}^{\mathbb{C}}\left(v_{2} \bar{\mu} \rho_{4}\right)+2 \rho_{4} v_{4}\right)\right)\right)\right] \overline{\left.f\right|_{k} \rho^{-1}} y^{k-w} \frac{d x d y}{y^{2}}
\end{aligned}
$$

Then we use Proposition 4.7 of [10] to expand $\phi_{\alpha, \beta}\left(z, P^{-1} t \mu \rho_{4}, v_{2}-P^{-1} t \mu \rho_{3}\right)$ $\left.\right|_{\alpha+2 \beta+1} \rho^{-1}$. We ignore convergence issues for the moment, and we shall address them 
later. When we compute the above integral term by term, then the essential part we have to consider is

$$
\begin{aligned}
& \int_{0}^{\infty} \int_{0}^{N}\left(\rho_{3}^{2} y\right)^{-\beta} L_{\beta}^{(\alpha)}\left(4 \pi y \mathbf{N}\left(\rho_{3} r-\rho_{4} v_{2}+v^{\prime}\right)\right) \\
& \quad e\left(2 \mathbf{N}\left(\rho_{3} r-\rho_{4} v_{2}+v^{\prime}\right) z\right) y^{-\gamma} e\left(i P^{-2} t^{2} \nu^{2} y^{-1}\right) \times \overline{\left.f\right|_{k} \rho^{-1}} y^{k-w} \frac{d x d y}{y^{2}} .
\end{aligned}
$$

Next we evaluate the integral with respect to $x$ using the Fourier expansion of $\left.f\right|_{k} \rho^{-1}$. In the case $\rho_{3} r-\rho_{4} v_{2}+v^{\prime} \neq 0$ this is equal to

$$
\begin{aligned}
& N \rho_{3}^{-2 \beta} \overline{a_{\rho}\left(N \mathbf{N}\left(\rho_{3} r-\rho_{4} v_{2}+v^{\prime}\right), s\right)}\left(4 \pi \mathbf{N}\left(\rho_{3} r-\rho_{4} v_{2}+v^{\prime}\right)\right)^{-k / 2} \\
& \quad \times \int_{0}^{\infty} y^{-\beta} L_{\beta}^{(\alpha)}\left(4 \pi \mathbf{N}\left(\rho_{3} r-\rho_{4} v_{2}+v^{\prime}\right) y\right) e^{-2 \pi \mathbf{N}\left(\rho_{3} r-\rho_{4} v_{2}+v^{\prime}\right) y} \\
& \quad \times y^{-\gamma} e^{-\pi P^{-2} t^{2} \nu^{2} y^{-1}} y^{-k / 2} W_{\frac{k}{2}, \bar{s}-\frac{1}{2}}\left(4 \pi \mathbf{N}\left(\rho_{3} r-\rho_{4} v_{2}+v^{\prime}\right) y\right) y^{k-2-w} d y .
\end{aligned}
$$

Using Lemma 4.8 of [10] we can evaluate the integral. Since there are exponentially decaying terms inside the integral, this converges regardless of $w$, so we can evaluate at $w=0$ to get

$$
\begin{aligned}
2 N & \rho_{3}^{-2 \beta} \overline{a_{\rho}\left(N \mathbf{N}\left(\rho_{3} r-\rho_{4} v_{2}+v^{\prime}\right), s\right)}\left(4 \pi \mathbf{N}\left(\rho_{3} r-\rho_{4} v_{2}+v^{\prime}\right)\right)^{-k / 2} \\
& \times \sum_{m=0}^{\beta} \sum_{e=0}^{\beta+\gamma-m}(-1)^{\beta+\gamma+e}\left(\begin{array}{c}
\beta+\alpha \\
\beta-m
\end{array}\right)\left(\begin{array}{c}
\beta+\gamma-m \\
e
\end{array}\right)(k / 2-s)_{\gamma+\beta-m-e}(m !)^{-1} \\
& \times\left(4 \pi \mathbf{N}\left(\rho_{3} r-\rho_{4} v_{2}+v^{\prime}\right)\right)^{m+(e+1) / 2}\left(\pi P^{-2} t^{2} \nu^{2}\right)^{m+(\alpha-\gamma+e) / 2} \\
& \times K_{2 \bar{s}-1-e}\left(4 \pi P^{-1} t \nu\left(\mathbf{N}\left(\rho_{3} r-\rho_{4} v_{2}+v^{\prime}\right)\right)\right.
\end{aligned}
$$

where $K$ is the $K$-Bessel function. In the case $\rho_{3} r-\rho_{4} v_{2}+v^{\prime}=0$ the integral is convergent for sufficiently large $\operatorname{Re}(w)$ and the result is

$$
\begin{aligned}
& N \rho_{3}^{-2 \beta}\left(\begin{array}{c}
\alpha+\beta \\
\beta
\end{array}\right)\left\{\overline{\tilde{a}_{\rho}(0, s)}\left(\pi P^{-2} t^{2} \nu^{2}\right)^{-\beta-\gamma+\frac{k}{2}-\bar{s}-w} \Gamma\left(w+\bar{s}-\frac{k}{2}+\gamma+\beta\right)\right. \\
& \left.+\overline{a_{\rho}(0, s)}\left(\pi P^{-2} t^{2} \nu^{2}\right)^{-\beta-\gamma+\frac{k}{2}+\bar{s}-1-w} \Gamma\left(w+1-\bar{s}-\frac{k}{2}+\beta+\gamma\right)\right\} .
\end{aligned}
$$

To justify this splitting of the integral, we need to justify the convergence for the change of order of integration and two infinite summations, i.e., sums over $t$ and $r$. If $\rho_{3} r-\rho_{4} v_{2}+v^{\prime}=0$, the sum over $t$ converges for sufficiently large $\operatorname{Re}(w)$. When 
$\rho_{3} r-\rho_{4} v_{2}+v^{\prime} \neq 0$, we have to study the convergence of

$$
\begin{aligned}
& \quad \sum_{\substack{t=1 \\
t \rho_{3}=-P v_{1}(\bmod M P)}} \sum_{r \in J}\left(P^{-1} t\right)^{\gamma} \tau^{\prime}\left(\rho^{-1}, P^{-1} t \mu \rho_{4}, v_{2}-P^{-1} t \mu \rho_{3}, v^{\prime}\right) e\left(-P^{-1} t\left(\operatorname{Tr}_{\mathbb{R}}^{\mathbb{C}}\left(v_{2} \bar{\mu} \rho_{4}\right)\right)\right) \\
& \times \int_{0}^{\infty} N y^{-\gamma} e\left(i P^{-2} t^{2} \nu^{2} y^{-1}\right) y^{-\beta} L_{\beta}^{(\alpha)}\left(4 \pi \mathbf{N}\left(\rho_{3} r-\rho_{4} v_{2}+v^{\prime}\right) y\right)\left(\rho_{3}^{-1}\left(\rho_{3} r-\rho_{4} v_{2}+v^{\prime}\right)\right)^{\alpha} \\
& \times e\left(2 \mathbf{N}\left(\rho_{3} r-\rho_{4} v_{2}+v^{\prime}\right) z+\operatorname{Tr}_{\mathbb{R}}^{\mathbb{C}}\left\{\left(2 r \rho_{3}-\rho_{4} v_{2}+v^{\prime}\right) \overline{\left(\rho_{2} v_{2}+P^{-1} t \mu\right)}\right\}\right) \\
& \times \overline{a_{\rho}\left(N \mathbf{N}\left(\rho_{3} r-\rho_{4} v_{2}+v^{\prime}\right), s\right)}\left|4 \pi \mathbf{N}\left(\rho_{3} r-\rho_{4} v_{2}+v^{\prime}\right) y\right|^{-\frac{k}{2}} \\
& \times W_{\frac{k}{2}, \bar{s}-\frac{1}{2}}\left(4 \pi \mathbf{N}\left(\rho_{3} r-\rho_{4} v_{2}+v^{\prime}\right) y\right) y^{k-2-w} d y .
\end{aligned}
$$

Then we see that summing first with taking absolute values and integrating gives a finite value, when we consider exponentially decaying terms $W_{\frac{k}{2}, s-\frac{1}{2}}\left(4 \pi \mathbf{N}\left(\rho_{3} r-\right.\right.$

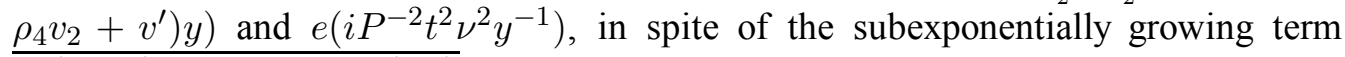
$\overline{a_{\rho}\left(N \mathbf{N}\left(\rho_{3} r-\rho_{4} v_{2}+v^{\prime}\right), s\right)}$. Therefore by Lebesgue's dominated convergence theorem we can carry out the previous term-by-term integration. The part corresponding to $\rho_{3} r-\rho_{4} v_{2}+v^{\prime}=0$ is simplified by using $\left(r-\rho_{3}^{-1} \rho_{4} v_{2}+\rho_{3}^{-1} v^{\prime}\right)^{\alpha}=0$ for $\alpha>0$ into

$$
\begin{aligned}
\delta_{\alpha, 0} & \frac{(k-1) ! P^{2 w-1} N}{2^{k-1}\left(\mathbf{N}(I)^{2} D\right)^{1 / 2}} i\left\{\nu^{2-2 \bar{s}-2 w} \pi^{-\frac{k}{2}+1-\bar{s}-w} q(k, s, w)\right. \\
& \times \sum_{\rho \in \mathcal{R}} \rho_{3}^{-1} \overline{\tilde{a}_{\rho}(0, s)} \tau(\rho) e\left(2 \rho_{4} \rho_{2} \mathbf{N}\left(v_{2}\right)\right) \sum_{\substack{t=1 \\
t \rho_{3} \equiv-P v_{1}(\bmod M P)}}\left(P^{-1} t\right)^{1-2 \bar{s}-2 w} e\left(-2 P^{-1} t \rho_{4} v_{4}\right) \\
& +\nu^{2 \bar{s}-2 w} \pi^{-\frac{k}{2}+\bar{s}-w} q(k, 1-s, w) \sum_{\rho \in \mathcal{R}} \rho_{3}^{-1} \overline{a_{\rho}(0, s)} \tau(\rho) e\left(2 \rho_{4} \rho_{2} \mathbf{N}\left(v_{2}\right)\right) \\
& \left.\times \sum_{\substack{t=1 \\
t \rho_{3} \equiv-P v_{1}(\bmod M P)}}\left(P^{-1} t\right)^{2 \bar{s}-1-2 w} e\left(-2 P^{-1} t \rho_{4} v_{4}\right)\right\}=\delta_{\alpha, 0} p(\nu, w) .
\end{aligned}
$$

Finally, the last part corresponding to $\rho_{3} r-\rho_{4} v_{2}+v^{\prime} \neq 0$ can be computed as in [10]. Note that rearranging the sum does not make a problem because it does not change the order between summations over $t$ and $r$, which are infinite sums. We arrive at the following simplified expression

$$
\begin{aligned}
C_{\alpha} & \sum_{\rho \in \mathcal{R}} \sum_{\substack{t=1 \\
t \rho_{3} \equiv-P v_{1}(\bmod M P)}} t^{-1} \sum_{r \in \bar{J}^{-1} \mathcal{D}_{K}^{-1}-0} \mathbf{N}(r)^{-k / 2}(r /|r|)^{\alpha} \\
& \times \overline{a_{\rho}(N \mathbf{N}(r), s)} \tau\left(\rho, r, v_{2}\right) e\left(2 P^{-1} t \operatorname{Tr}_{\mathbb{R}}^{\mathbb{C}}(\bar{r} \mu)-\rho_{4} v_{4}\right) q_{\alpha}\left(2 \pi P^{-1} t \nu|r|, \lambda\right),
\end{aligned}
$$

which completes the proof in the $\alpha \geq 0$ case. The last equality follows from the fact that $v_{2} \in \bar{J}^{-1} \mathcal{D}_{K}^{-1}$. Finally, since the relationship between $\alpha \geq 0$ and $\alpha \leq 0$ is simply a restatement of Theorem 3.3 (2), this finishes the proof of Theorem 4.2. 
We can simplify Theorem 4.2 by applying the Doi-Naganuma lifting with character defined in the section 3 of [10] and mixing in characters as in [10, Theorem 4.2]. For a character $\chi(\bmod N)$, let

$W M F_{k,-\lambda, \chi}^{+}\left(\Gamma_{0}(N)\right):=\left\{f \in W M F_{k,-\lambda}^{+}(\Gamma(N))|f|_{k} \sigma=\chi(d) f\right.$ for all $\left.\sigma \in \Gamma_{0}(N)\right\}$.

We define

$$
\begin{aligned}
\widehat{p}(\nu, w)= & (k-1) ! N \#\left\{\rho \in \mathcal{R} \mid\left(\rho_{3}, N\right)=1\right\} \\
& \times\left(\overline{\tilde{a}_{S}(0, s)} r_{k, D \mathbf{N}(I)^{2}}(s+w)+\overline{a_{S}(0, s)} r_{k, D \mathbf{N}(I)^{2}}(1-s+w)\right), \\
r_{k, D \mathbf{N}(I)^{2}}(s)= & \nu^{2-2 \bar{s}} \sum_{\beta, \gamma} \beta !^{-1} \gamma !^{-1} i^{\gamma-1} 2^{-2 \beta}\left(D \mathbf{N}(I)^{2}\right)^{2 \beta+\frac{1}{2}} \\
& \times \Gamma\left(\bar{s}+\frac{k-1+\gamma}{2}\right) \sum_{t=1}^{\infty} \chi \chi_{-D}(t) t^{1-2 \bar{s}}
\end{aligned}
$$

Here $S=\left(\begin{array}{cc}0 & -1 \\ 1 & 0\end{array}\right)$.

Theorem 4.3. For $P=1$ and $M=N$ the Doi-Naganuma lifting with character of $f$ in $W M F_{k,-\lambda, \chi}^{+}\left(\Gamma_{0}(N)\right)$ is given by the constant term of the Laurent expansion at $w=0$ of the analytic continuation of

$$
\begin{aligned}
& F_{\alpha}^{r e g}(g(\mu, \nu) ; f) \\
= & C_{\alpha}^{\prime} \sum_{t=1}^{\infty} t^{-1} \chi \chi_{-D}(t) \sum_{r \in J-0}|r|^{-k}(r /|r|)^{\alpha} \overline{b\left(N \mathbf{N}(r) / D \mathbf{N}(I)^{2}\right)} \\
& \times q_{|\alpha|}\left(2 \pi \nu t|r| /\left[D \mathbf{N}(I)^{2}\right]^{1 / 2}, \lambda\right) e\left(2 \operatorname{Tr}_{\mathbb{R}}^{\mathbb{C}}\left(r \partial^{-1} \mathbf{N}(I)^{-1} t \bar{\mu}\right)\right)+\delta_{\alpha, 0} \widehat{p}(\nu, w) .
\end{aligned}
$$

Here $\partial \in \mathfrak{H}$ is the purely imaginary generator of $\mathcal{D}_{K}, \chi \chi_{-D}$ is a (not necessarily primitive) character $(\bmod N)$ and

$$
C_{\alpha}^{\prime}=C_{|\alpha|}\left[D \mathbf{N}(I)^{2} / N\right]^{k / 2} N i^{2-\alpha} \#\left\{\rho \in \mathcal{R} \mid\left(\rho_{3}, N\right)=1\right\} .
$$

\section{ACKNOWLEDGMENT}

The authors thank YoungJu Choie for suggesting to investigate the extension of the regularized Doi-Naganuma lifting. She also gave us many valuable comments that improved this paper a lot. We also thank the referee for considerably helping us to make the paper readable. Subong Lim was supported by Samsung Science and Technology Foundation under Project SSTF-BA1301-11. Seokho Jin was supported by the National Research Foundation of Korea (NRF) grant funded by the Korea government (MSIP) (No. 2014001824). 


\section{REFERENCES}

1. T. Asai, On the Doi-Naganuma lifting associated with imaginary quadratic fields, Nagoya Math. J., 71 (1978), 149-167.

2. R. Borcherds, Automorphic forms with singularities on Grassmannians, Invent. Math. 132 (1998), 491-562.

3. K. Bringmann and K. Ono, Dyson's ranks and Maass forms, Ann. of Math., 171(2) (2010), 419-449.

4. K. Bringmann and D. Penniston, Arithmetic properties of non-harmonic weak Maass forms, Proc. Amer. Math. Soc., 137 (2009), 825-833.

5. J. H. Bruinier, Borcherds products on $O(2, l)$ and Chern classes of Heegner divisors, Lecture Notes in Math., 1780 (2002).

6. J. H. Bruinier and J. Funke, Traces of CM values of modular functions, J. Reine Angew. Math., 594 (2006), 1-33.

7. J. H. Bruinier and K. Ono, Heegner divisors, L-functions, and Maass forms, Ann. of Math., 172(2) (2010), 2135-2181.

8. B. A. Cipra, On the Niwa-Shintani theta-kernel lifting of modular forms, Nagoya Math. J., 91 (1983), 49-117.

9. K. Doi and H. Naganuma, On the functional equation of certain Dirichlet series, Invent. Math. 9 (1969), 1-14.

10. S. Friedberg, On Maass wave forms and the imaginary quadratic Doi-Naganuma lifting, Math. Ann., 263 (1983), 483-508.

11. S. Friedberg, On the imaginary quadratic Doi-Naganuma lifting of modular forms of arbitrary level, Nagoya Math. J., 92 (1983), 1-20.

12. S. Friedberg, Differential operators and theta series, Trans. Amer. Math. Soc., 287 (1985), 569-589.

13. J. Harvey and G. Moore, Algebras, BPS states, and strings, Nuclear Phys. B, 463(2-3) (1996), 315-368.

14. E. Hecke, Mathematische Werke, Vandenhoeck \& Ruprecht, Gottingen, 1970, No. 14.

15. S. Husseini and M. Knopp, Eichler cohomology and automorphic forms, Illinois J. Math., 15 (1971), 565-577.

16. S. Katok and P. Sarnak, Heegner points, cycles, and Maass forms, Israel J. Math., 84 (1984), 193-227.

17. S. Kudla, Derivatives of Eisenstein series and arithmetic geometry, Proceedings of the International Congress of Mathematicians, Vol. II (Beijing, 2002), Higher ed. Press, Beijing, 2002, pp. 173-183.

18. S. Kudla and J. Millson, Intersection numbers of cycles on locally symmetric spaces and Fourier coefficients of holomorphic modular forms in several complex variables, Inst. Hautes Études Sci. Publ. Math., 71 (1990), 121-172. 
19. S. Kudla, M. Rapoport and T. Yang, Derivatives of Eisenstein series and Faltings heights, Compos. Math., 140 (2004), 887-951.

20. H. Naganuma, On the coincidence of two Dirichlet series associated with cusp forms of Hecke's "Neben"-type and Hilbert modular forms over a real quadratic field, J. Math. Soc. Japan, 25 (1967), 547-555.

21. T. Shintani, On construction of holomorphic cusp forms of half integral weight, Nagoya Math. J., 58 (1975), 83-126.

22. E. T. Whittaker and G. N. Watson, A Course of Modern Analysis, (4ed. CUP).

23. D. Zagier, Traces of singular moduli, in: Motives, Polylogarithms and Hodge Theory, Part I, F. Bogomolov and L. Katzarkov, eds., International Press, Somerville, 2002.

24. D. Zagier, Ramanujan's mock theta functions and their applications [d'aprés Zwegers and Bringmann-Ono], Séminaire Bourbaki, 60éme année, 2006-2007, no. 986.

25. S. Zwegers, Mock Theta Functions, Ph.D. Thesis, U. Utrecht, 2002.

Seokho Jin

Department of Mathematical Sciences and ASARC

Korea Advanced Institute of Science and Technology

Daejeon 305-701

Republic of Korea

E-mail: seokho.jin1@gmail.com

Subong Lim

School of Mathematics

Korea Institute for Advanced Study

Hoegiro 85, Dongdaemun-gu, Seoul 130-722

Republic of Korea

E-mail: subong@kias.re.kr 